\title{
Local Modelling with A Priori Known Bounds Using Direct Weight Optimization
}

\author{
Jacob Roll, Alexander Nazin, Lennart Ljung \\ Division of Automatic Control \\ Department of Electrical Engineering \\ Linköpings universitet, SE-581 83 Linköping, Sweden \\ WWW: http://www. control.isy.liu.se \\ E-mail: roll,ljung@isy.liu.se, nazine@ipu.rssi.ru
}

January 9, 2003

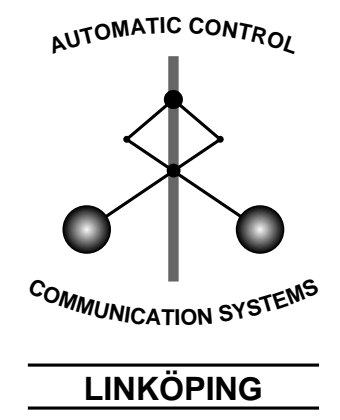

Report no.: LiTH-ISY-R-2484

Submitted to ECC'03

Technical reports from the Control \& Communication group in Linköping are available at http://www. control.isy.liu.se/publications. 



\title{
Local Modelling with A Priori Known Bounds Using Direct Weight Optimization
}

\author{
Jacob Roll;, Alexander Nazin†, Lennart Ljung*
}

January 9, 2003

\begin{abstract}
In local modelling, function estimates are computed from observations in a local neighborhood of the point of interest. A central question is how to choose the size of the neighborhood. Often this question has been tackled using asymptotic (in the number of observations) arguments. The recently introduced direct weight optimization approach is a non-asymptotic approach, minimizing an upper bound on the mean-square error. In this paper the approach is extended to also take a priori known bounds on the function and its derivative into account. It is shown that the result will sometimes, but not always, be improved by this information. The proposed approach can be applied, e.g., to prediction of nonlinear dynamic systems and model predictive control.
\end{abstract}

Keywords: Local modelling, non-parametric identification, nonlinear systems, minimax techniques, convex programming

\section{Introduction}

Local modelling of different types have been of great interest for a long time in system identification and statistics. A local model or method for function approximation or prediction computes the estimate using information from observations in a local neighborhood of the point of interest. Many of the different nonlinear black-box methods are of this type, such as radial basis neural networks, multiple-model approaches etc. (see, e.g., [4,9]). In statistics, local methods such as kernel methods [5,11], local polynomial modelling [3], and trees [2] have been popular.

A central question in local modelling is the bandwidth question: how to select the size of the neighborhood. This becomes a bias/variance trade-off, which has been studied extensively in the statistics literature, and many solutions based on asymptotic (in the number of observations) arguments have been proposed.

In this paper, a direct weight optimization (DWO) approach is considered. This approach is a non-asymptotic approach, where the weights of a linear (or affine) estimator are determined by minimizing a uniform upper bound on the

\footnotetext{
*Div. of Automatic Control, Linköping University, SE-58183 Linköping, Sweden, e-mail: roll, ljung@isy.liu.se

$\dagger$ Institute of Control Sciences, Profsoyuznaya str., 65, 117997 Moscow, Russia, e-mail: nazine@ipu.rssi.ru
} 
mean-square error (MSE) over function classes having a Lipshitz bound $L$ on the derivative. It turns out that the optimization problem can be formulated as a quadratic program (QP) or a second-order cone program (SOCP). The basic idea was first presented in [6] and [8], where it was also shown that the approach has several interesting properties:

- Outside a certain bandwidth, the weights will automatically become zero. This is referred to as automatic bandwidth selection.

- Asymptotically (as $N \rightarrow \infty, N$ being the number of observations), the weights will (under certain assumptions) converge to the weights obtained by local linear modelling with an asymptotically optimal kernel function (see [3]).

- When $L=0$, the function estimates obtained are the same as for a global "affine ARX" model.

Here, the approach is extended to also take a priori known bounds on the function and its derivative into account. In practice, such bounds may be given by physical constraints, or by practical experience and expert knowledge. It turns out that the extension is very natural, and some theorems can be given about when we actually benefit from the extra information.

In Section 2, the basic problem is presented for the scalar case, and in Sections 3 and 4 the DWO approach is outlined. Section 5 deals with multivariate functions, and conclusions in Section 6 .

\section{Basic problem}

Let us consider the problem of estimating the value $f\left(\varphi_{0}\right)$ of an unknown function $f: \mathbf{R} \rightarrow \mathbf{R}$ at a given point $\varphi_{0}$, given a set of input-output pairs $\left\{\left(\varphi_{k}, y_{k}\right)\right\}_{k=1}^{N}$, coming from the relation

$$
y_{k}=f\left(\varphi_{k}\right)+e_{k}
$$

Assume that the function $f$ is continuously differentiable, and that there are known positive constants $L, \delta, \Delta$, and known constants $a, b$ such that

$$
\begin{aligned}
\left|f^{\prime}\left(\varphi_{1}\right)-f^{\prime}\left(\varphi_{2}\right)\right| & \leq L\left|\varphi_{1}-\varphi_{2}\right| \quad \forall \varphi_{1}, \varphi_{2} \in \mathbf{R} \\
\left|f\left(\varphi_{0}\right)-a\right| & \leq \delta \\
\left|f^{\prime}\left(\varphi_{0}\right)-b\right| & \leq \Delta .
\end{aligned}
$$

Denote the class of functions satisfying these assumptions by $\mathcal{F}_{2}(L, \delta, \Delta)$.

The noise terms $e_{k}$ are independent random variables with $E e_{k}=0$ and $E e_{k}^{2}=\sigma_{k}^{2}$ where $\sigma_{k}$ are assumed to be positive constants, given a priori. For simplicity, only constant variance (i.e., $\sigma_{k}^{2}=\sigma^{2}$ ) will be considered in this paper. The notation

$$
\tilde{\varphi}_{k}=\varphi_{k}-\varphi_{0}
$$

and $X=\left(\varphi_{1}, \ldots, \varphi_{N}\right)$ will also be used.

There are some particular cases that deserve special attention: 
- If $\delta \rightarrow+\infty$ then the limit class

$$
\mathcal{F}_{2}(L, \Delta) \triangleq \mathcal{F}_{2}(L, \Delta, \infty)=\left.\bigcup_{t=1}^{\infty} \mathcal{F}_{2}(L, \delta, \Delta)\right|_{\delta=t}
$$

describes the situation where we have no direct a priori information on function value $f\left(\varphi_{0}\right)$.

- If in addition $\Delta \rightarrow+0$, then the limit class $\mathcal{F}_{2}(L, 0)$ represents a set of functions meeting condition (2) and having a given derivative $f^{\prime}\left(\varphi_{0}\right)=b$.

- If both $\delta \rightarrow+\infty$ and $\Delta \rightarrow+\infty$ then the limit class

$$
\mathcal{F}_{2}(L) \triangleq \mathcal{F}_{2}(L, \infty)=\left.\bigcup_{t=1}^{\infty} \mathcal{F}_{2}(L, \Delta)\right|_{\Delta=t}
$$

represents a set of continuously differentiable functions meeting the only condition (2). This case was studied previously in $[6,8]$.

A common approach for the given estimation problem is to use a linear estimator

$$
\hat{f}\left(\varphi_{0}\right)=\sum_{k=1}^{N} w_{k} y_{k}
$$

The problem then reduces to finding good weights $w_{k}$, according to some criterion. In the following, we will consider the slightly more general class of affine estimators:

$$
\hat{f}\left(\varphi_{0}\right)=w_{0}+\sum_{k=1}^{N} w_{k} y_{k}
$$

The performance of an estimator $\hat{f}\left(\varphi_{0}\right)$ will be evaluated by the worst-case mean-square error (MSE) defined by

$$
V\left(\mathcal{F}, w_{0}, w\right)=\sup _{f \in \mathcal{F}} M S E\left(f, w_{0}, w\right)
$$

where

$$
\operatorname{MSE}\left(f, w_{0}, w\right)=E\left[\left(\hat{f}\left(\varphi_{0}\right)-f\left(\varphi_{0}\right)\right)^{2} \mid X\right]
$$

In Section 3, upper bounds on $V\left(\mathcal{F}, w_{0}, w\right)$ are given for the different function classes defined above. As it turns out, these upper bounds can then be minimized using quadratic programming (QP), yielding optimal (in this sense) estimators.

\section{A non-asymptotic approach}

\subsection{Class $\mathcal{F}=\mathcal{F}_{2}(L, \delta, \Delta)$}

Let us again consider the affine estimator $(9)$ and the function class $\mathcal{F}_{2}(L, \delta, \Delta)$ for finite $\delta, \Delta$. For this estimator and class, the worst-case MSE (10) has the 
following upper bound:

$$
\begin{aligned}
& V\left(\mathcal{F}_{2}(L, \delta, \Delta), w_{0}, w\right) \leq U_{0}\left(w_{0}, w\right) \\
& =\left(\left|w_{0}+a\left(\sum_{k=1}^{N} w_{k}-1\right)+b \sum_{k=1}^{N} w_{k} \widetilde{\varphi}_{k}\right|+\delta\left|\sum_{k=1}^{N} w_{k}-1\right|\right. \\
& \left.\quad+\Delta\left|\sum_{k=1}^{N} w_{k} \widetilde{\varphi}_{k}\right|+\frac{L}{2} \sum_{k=1}^{N}\left|w_{k}\right| \widetilde{\varphi}_{k}^{2}\right)^{2}+\sigma^{2} \sum_{k=1}^{N} w_{k}^{2}
\end{aligned}
$$

This is true, since for any function $f \in \mathcal{F}_{2}(L, \delta, \Delta)$ the estimation error may be represented as follows

$$
\begin{aligned}
\hat{f}\left(\varphi_{0}\right)-f\left(\varphi_{0}\right)= & w_{0}+a\left(\sum_{k=1}^{N} w_{k}-1\right)+b \sum_{k=1}^{N} w_{k} \widetilde{\varphi}_{k} \\
& +\left(f\left(\varphi_{0}\right)-a\right)\left(\sum_{k=1}^{N} w_{k}-1\right)+\left(f^{\prime}\left(\varphi_{0}\right)-b\right) \sum_{k=1}^{N} w_{k} \widetilde{\varphi}_{k} \\
& +\sum_{k=1}^{N} w_{k}\left(f\left(\varphi_{k}\right)-f\left(\varphi_{0}\right)-f^{\prime}\left(\varphi_{0}\right) \widetilde{\varphi}_{k}\right)+\sum_{k=1}^{N} w_{k} e_{k}
\end{aligned}
$$

Due to a well known lemma, the inequality

$$
\left|f\left(\varphi_{k}\right)-f\left(\varphi_{0}\right)-f^{\prime}\left(\varphi_{0}\right) \widetilde{\varphi}_{k}\right| \leq \frac{L}{2} \widetilde{\varphi}_{k}^{2}
$$

follows from (2). Now, the MSE (11) satisfies

$$
\begin{aligned}
\operatorname{MSE}\left(f, w_{0}, w\right) \leq & \left(\left|w_{0}+a\left(\sum_{k=1}^{N} w_{k}-1\right)+b \sum_{k=1}^{N} w_{k} \widetilde{\varphi}_{k}\right|\right. \\
& +\left|f\left(\varphi_{0}\right)-a\right| \cdot\left|\sum_{k=1}^{N} w_{k}-1\right|+\left|f^{\prime}\left(\varphi_{0}\right)-b\right| \cdot\left|\sum_{k=1}^{N} w_{k} \widetilde{\varphi}_{k}\right| \\
& \left.+\sum_{k=1}^{N}\left|w_{k}\right| \cdot\left|f\left(\varphi_{k}\right)-f\left(\varphi_{0}\right)-f^{\prime}\left(\varphi_{0}\right) \widetilde{\varphi}_{k}\right|\right)^{2}+\sigma^{2} \sum_{k=1}^{N} w_{k}^{2}
\end{aligned}
$$

from which the upper bound (12) follows directly.

Note that the upper bound $U_{0}\left(w_{0}, w\right)$ is easily minimized with respect to $w_{0}$ for any $w \in \mathbf{R}^{N}$. Indeed,

$$
\arg \min _{w_{0}} U_{0}\left(w_{0}, w\right)=-a\left(\sum_{k=1}^{N} w_{k}-1\right)-b \sum_{k=1}^{N} w_{k} \widetilde{\varphi}_{k}
$$

Thus, we arrive at the following consequence: For the function class $\mathcal{F}_{2}(L, \delta, \Delta)$, the affine estimator minimizing $U_{0}\left(w_{0}, w\right)$ may be sought among the estimators satisfying

$$
\hat{f}\left(\varphi_{0}\right)=\sum_{k=1}^{N} w_{k} y_{k}-a\left(\sum_{k=1}^{N} w_{k}-1\right)-b \sum_{k=1}^{N} w_{k} \widetilde{\varphi}_{k}, \quad w \in \mathbf{R}^{N}
$$


For this kind of estimators, $V\left(\mathcal{F}_{2}(L, \delta, \Delta), w_{0}, w\right)$ has the following upper bound, which follows directly from (12) and (16):

$$
\begin{aligned}
& V\left(\mathcal{F}_{2}(L, \delta, \Delta), w_{0}, w\right) \leq U_{0}(w) \\
& \quad=\left(\delta\left|\sum_{k=1}^{N} w_{k}-1\right|+\Delta\left|\sum_{k=1}^{N} w_{k} \widetilde{\varphi}_{k}\right|+\frac{L}{2} \sum_{k=1}^{N}\left|w_{k}\right| \widetilde{\varphi}_{k}^{2}\right)^{2}+\sigma^{2} \sum_{k=1}^{N} w_{k}^{2}
\end{aligned}
$$

Let us take a closer look at (13). It is easy to see, that if $\delta \rightarrow \infty$, the MSE might be arbitrarily large unless $\sum_{k=1}^{N} w_{k}=1$, since the term

$$
\left(f\left(\varphi_{0}\right)-a\right)\left(\sum_{k=1}^{N} w_{k}-1\right)
$$

is unbounded. In fact, we can show the following interesting theorem:

Theorem 3.1. Assume that $\widetilde{\varphi}_{k} \neq 0, k=1, \ldots, N$. Given $a, b \in \mathbf{R}$ and $L, \Delta \in$ $(0,+\infty)$, there exists a $\delta_{0} \in(0,+\infty)$ such that for any $\delta>\delta_{0}$, the minimum of the upper bound $U_{0}(w)$ given by (18) with respect to $w \in \mathbf{R}^{N}$ is attained on the subspace

$$
\sum_{k=1}^{N} w_{k}=1
$$

and does not depend on $\delta$. In other words, given a sufficiently large $\delta$, the affine estimator (9) minimizing $U_{0}\left(w_{0}, w\right)$ can be found in the form

$$
\hat{f}\left(\varphi_{0}\right)=\sum_{k=1}^{N} w_{k} y_{k}-b \sum_{k=1}^{N} w_{k} \widetilde{\varphi}_{k}, \quad \sum_{k=1}^{N} w_{k}=1
$$

with the weights $\left(w_{1}, \ldots, w_{N}\right)$ minimizing the simpler upper bound

$$
U_{1}(w)=\left(\Delta\left|\sum_{k=1}^{N} w_{k} \widetilde{\varphi}_{k}\right|+\frac{L}{2} \sum_{k=1}^{N}\left|w_{k}\right| \widetilde{\varphi}_{k}^{2}\right)^{2}+\sigma^{2} \sum_{k=1}^{N} w_{k}^{2}
$$

subject to the constraint (19).

Proof. See [7].

\subsection{Class $\mathcal{F}=\mathcal{F}_{2}(L, \Delta)$}

Let us now turn to class $\mathcal{F}_{2}(L, \Delta)$, i.e., the case $\delta \rightarrow \infty$. From the remark just before Theorem 3.1, it follows that the MSE cannot be bounded above unless $\sum_{k=1}^{N} w_{k}=1$. On the other hand, if this requirement is satisfied, we get the following upper bound on the worst-case MSE, which can be shown analogously to $(12)$ :

$$
\begin{aligned}
& V\left(\mathcal{F}_{2}(L, \Delta), w_{0}, w\right) \leq U_{1}\left(w_{0}, w\right) \\
& \quad=\left(\left|w_{0}+b \sum_{k=1}^{N} w_{k} \widetilde{\varphi}_{k}\right|+\Delta\left|\sum_{k=1}^{N} w_{k} \widetilde{\varphi}_{k}\right|+\frac{L}{2} \sum_{k=1}^{N}\left|w_{k}\right| \widetilde{\varphi}_{k}^{2}\right)^{2}+\sigma^{2} \sum_{k=1}^{N} w_{k}^{2}
\end{aligned}
$$


By minimizing the upper bound with respect to $w_{0}$, it can be seen that the minimizing estimator will be in the form (20), and that $w$ can be found by minimizing (21) under assumption (19).

Analogously to Theorem 3.1 , we can study what happens when $\Delta$ is large.

Theorem 3.2. Suppose that $\widetilde{\varphi}_{k} \neq 0, k=1, \ldots, N$. Given $L \in(0,+\infty)$, there exists a $\Delta_{0} \in(0,+\infty)$ such that for any $\Delta>\Delta_{0}$, the minimum of the upper bound $U_{1}(w)$ given by (21), subject to the constraint (19), is attained on the subspace

$$
\sum_{k=1}^{N} w_{k}=1, \quad \sum_{k=1}^{N} w_{k} \widetilde{\varphi}_{k}=0
$$

and does not depend on $\Delta$. In other words, given a sufficiently large $\Delta$, the affine estimator (9) minimizing $U_{1}\left(w_{0}, w\right)$ can be found in the form (8) by minimizing the upper bound

$$
U_{2}(w)=\left(\frac{L}{2} \sum_{k=1}^{N}\left|w_{k}\right| \widetilde{\varphi}_{k}^{2}\right)^{2}+\sigma^{2} \sum_{k=1}^{N} w_{k}^{2}
$$

subject to constraints (23).

Proof. See [7].

\subsection{Class $\mathcal{F}=\mathcal{F}_{2}(L)$}

For class $\mathcal{F}_{2}(L)$, following a similar line of argument as for $\mathcal{F}_{2}(L, \Delta)$, we can see that a finite MSE can be guaranteed only if the weights $w$ satisfy (23). Under this requirement, on the other hand, we get the following upper bound on the worst-case MSE:

$$
V\left(\mathcal{F}_{2}(L), w_{0}, w\right) \leq U_{2}\left(w_{0}, w\right)=\left(\left|w_{0}\right|+\frac{L}{2} \sum_{k=1}^{N}\left|w_{k}\right| \widetilde{\varphi}_{k}^{2}\right)^{2}+\sigma^{2} \sum_{k=1}^{N} w_{k}^{2}
$$

Hence, by minimizing the upper bound with respect to $w_{0}$, we will obtain a minimizing estimator in the form (8), where $w$ can be found by minimizing (24) subject to the constraints (23).

\section{QP formulation}

In Section 3, it was pointed out how the weights $w_{0}$ and $w_{k}$ of the affine estimator (9) could be chosen by minimizing different expressions, in order to get estimators with a guaranteed upper bound on the worst-case MSE. In this section we will show that these minimization problems can be formulated as convex quadratic programs (QP) (see $[1])$.

To begin with, let us consider the function class $\mathcal{F}_{2}(L, \delta, \Delta)$ and the problem of finding the affine estimator minimizing (18). 
Theorem 4.1. Given the positive numbers $\delta, \Delta, L$, consider the following minimization problem:

$$
\begin{aligned}
\min _{w, s} & \left(\delta s_{a}+\Delta s_{b}+\frac{L}{2} \sum_{k=1}^{N} \widetilde{\varphi}_{k}^{2} s_{k}\right)^{2}+\sigma^{2} \sum_{k=1}^{N} s_{k}^{2} \\
\text { subj. to } \quad & s_{a} \geq \pm\left(\sum_{k=1}^{N} w_{k}-1\right) \\
& s_{b} \geq \pm \sum_{k=1}^{N} w_{k} \widetilde{\varphi}_{k} \\
& s_{k} \geq \pm w_{k}, \quad k=1, \ldots, N
\end{aligned}
$$

where $w=\left(w_{1}, \ldots, w_{N}\right)$ and $s=\left(s_{a}, s_{b}, s_{1}, \ldots, s_{N}\right)$. Then $w^{*}$ is a minimizer of (18) if and only if there is a vector $s^{*}$ such that $\left(w^{*}, s^{*}\right)$ is a minimizer of (26).

Proof. Given a feasible solution $w$ to (18), we can get a feasible solution to (26) with the same value of the objective function by using the same $w$ and

$$
\begin{aligned}
s_{a} & =\left|\sum_{k=1}^{N} w_{k}-1\right| \\
s_{b} & =\left|\sum_{k=1}^{N} w_{k} \widetilde{\varphi}_{k}\right| \\
s_{k} & =\left|w_{k}\right|, \quad k=1, \ldots, N
\end{aligned}
$$

Hence (26) is a relaxation of (18), and it suffices to show that for a minimizer $\left(w^{*}, s^{*}\right)$ of $(26),(27)$ will hold. Suppose, e.g., that $s_{1}^{*}>\left|w_{1}^{*}\right|$. Then, without changing any other variables, the value of the objective function can be reduced by decreasing $s_{1}^{*}$. This can be seen by observing that the coefficient before $s_{1}^{*}$ is non-negative in the first sum of the objective function, and positive in the second sum of the objective function, so decreasing $s_{1}^{*}$ will decrease at least one of these sums, and hence the objective function. Hence, $s_{1}^{*}=\left|w_{1}^{*}\right|$. By similar arguments, one can show that the other equalities of (27) will also hold at the optimum, and the theorem is proven.

Remark 4.1. Note that (26) is a convex QP and can therefore be solved efficiently.

Starting from Theorem 4.1, we can now formulate QP:s for all the other cases mentioned in Section 3. Since the constraints (19) and (23) are all linear in $w$, they can just be added to the QP.

\section{$5 \quad$ Estimating multivariate functions}

In this paper, we have so far assumed that the function to be estimated has a scalar argument. In most applications, in particular to dynamic systems, the regressors will have a higher dimension. The extension to this case is immediate. In this section, we will describe some of the aspects of this kind of extension. 
We now consider the problem of estimating the value $f\left(\varphi_{0}\right)$ of an unknown multivariate, continuously differentiable function $f: \mathbf{R}^{n} \rightarrow \mathbf{R}$ at a given point $\varphi_{0}$, given a set of input-output pairs $\left\{\left(\varphi_{k}, y_{k}\right)\right\}_{k=1}^{N}$, coming from the relation

$$
y_{k}=f\left(\varphi_{k}\right)+e_{k}
$$

Instead of the assumptions (2)-(4), we make the following assumptions:

$$
\begin{aligned}
\left\|\nabla f\left(\varphi_{1}\right)-\nabla f\left(\varphi_{2}\right)\right\| & \leq L\left\|\varphi_{1}-\varphi_{2}\right\| \quad \forall \varphi_{1}, \varphi_{2} \in \mathbf{R}^{n} \\
\left|f\left(\varphi_{0}\right)-a\right| & \leq \delta \\
\left\|\nabla f\left(\varphi_{0}\right)-b\right\| & \leq \Delta .
\end{aligned}
$$

With some abuse of notation, we let $\mathcal{F}_{2}(L, \delta, \Delta), \mathcal{F}_{2}(L, \Delta)$, and $\mathcal{F}_{2}(L)$ denote also their multivariate counterparts. For $\mathcal{F}_{2}(L, \delta, \Delta)$ and an affine estimator (9), the worst-case MSE (10) has the following upper bound, which is similar to the scalar case:

$$
\begin{aligned}
& V\left(\mathcal{F}_{2}(L, \delta, \Delta), w_{0}, w\right) \leq U_{0}\left(w_{0}, w\right)\left(\left|w_{0}+a\left(\sum_{k=1}^{N} w_{k}-1\right)+b^{T} \sum_{k=1}^{N} w_{k} \widetilde{\varphi}_{k}\right|+\delta\left|\sum_{k=1}^{N} w_{k}-1\right|\right. \\
&\left.+\Delta\left\|\sum_{k=1}^{N} w_{k} \widetilde{\varphi}_{k}\right\|+\frac{L}{2} \sum_{k=1}^{N}\left|w_{k}\right|\left\|\widetilde{\varphi}_{k}\right\|^{2}\right)^{2}+\sigma^{2} \sum_{k=1}^{N} w_{k}^{2}
\end{aligned}
$$

As in the scalar case, we can immediately eliminate $w_{0}$ by minimizing (32) for an arbitrary $w$, giving that the affine estimator minimizing $V\left(\mathcal{F}_{2}(L, \delta, \Delta), w_{0}, w\right)$ may be sought among the estimators satisfying

$$
\hat{f}\left(\varphi_{0}\right)=\sum_{k=1}^{N} w_{k} y_{k}-a\left(\sum_{k=1}^{N} w_{k}-1\right)-b^{T} \sum_{k=1}^{N} w_{k} \widetilde{\varphi}_{k}, \quad w \in \mathbf{R}^{N}
$$

For this kind of estimators, the worst-case MSE (10) over the class $\mathcal{F}_{2}(L, \delta, \Delta)$ has the following upper bound:

$$
\begin{aligned}
& V\left(\mathcal{F}_{2}(L, \delta, \Delta), w_{0}, w\right) \leq U_{0}(w) \\
& =\left(\delta\left|\sum_{k=1}^{N} w_{k}-1\right|+\Delta\left\|\sum_{k=1}^{N} w_{k} \widetilde{\varphi}_{k}\right\|+\frac{L}{2} \sum_{k=1}^{N}\left|w_{k}\right|\left\|\widetilde{\varphi}_{k}\right\|^{2}\right)^{2}+\sigma^{2} \sum_{k=1}^{N} w_{k}^{2}
\end{aligned}
$$

So far, the differences to the scalar case have been small and obvious. However, when trying to transform the problem of minimizing (34) into a standard convex optimization problem, it turns out that it is impossible to formulate it as a QP problem. What prohibits this is the term $\Delta\left\|\sum_{k=1}^{N} w_{k} \widetilde{\varphi}_{k}\right\|$ which is the norm of a linear combination of vectors. Instead, we can formulate the problem as a second-order cone program (SOCP), which is another standard class of convex optimization problems (see [1]). To do this, we introduce some slack 
variables $s=\left(\begin{array}{lll}s_{1} & \ldots & s_{N}\end{array}\right)^{T}$ and $t=\left(t_{a} t_{b} t_{c}\right)^{T}$, and get

$$
\begin{array}{ll}
\min _{w, s, t} & t_{c} \\
\text { subj. to } & \left(\delta t_{a}+\Delta t_{b}+\frac{L}{2} \sum_{k=1}^{N}\left\|\widetilde{\varphi}_{k}\right\|^{2} s_{k}\right)^{2}+\sigma^{2} \sum_{k=1}^{N} s_{k}^{2} \leq t_{c} \\
& \left|\sum_{k=1}^{N} w_{k}-1\right| \leq t_{a} \\
& || \sum_{k=1}^{N} w_{k} \widetilde{\varphi}_{k}|| \leq t_{b} \\
& \left|w_{k}\right| \leq s_{k}, \quad k=1, \ldots, N
\end{array}
$$

This problem is in standard SOCP form, except for the first, quadratic constraint. However, straightforward calculations show that this constraint is equivalent to

$$
\left\|\left(\begin{array}{c}
2\left(\delta t_{a}+\Delta t_{b}+\frac{L}{2} \sum_{k=1}^{N}\left\|\widetilde{\varphi}_{k}\right\|^{2} s_{k}\right) \\
2 \sigma s \\
1-t_{c}
\end{array}\right)\right\| \leq 1+t_{c}
$$

thus completing the problem reformulation.

For the other function classes, $\mathcal{F}_{2}(L, \Delta)$ and $\mathcal{F}_{2}(L)$, the extension to the multivariate case is done completely similarly. The minimization problem for $\mathcal{F}_{2}(L, \Delta)$ will also yield a SOCP, while the minimization problem for $\mathcal{F}_{2}(L)$ will still be possible to express as a $\mathrm{QP}$, since the term $\Delta\left\|\sum_{k=1}^{N} w_{k} \widetilde{\varphi}_{k}\right\|$ vanishes.

\section{Conclusions}

In this paper, a non-asymptotic approach for local modelling has been presented. It was shown that the approach is easily extended to the case when a priori bounds on the function and its derivative are known. Theorems 3.1 and 3.2 however showed that when the bounds are very wide, the extra information may not be enough to improve the solution.

The application of these methods to dynamical systems with the regressors $\varphi_{k}$ being built up by past inputs and outputs is straightforward. The method can be used as an alternative to building non-linear black-box models in a "Model-On-Demand" fashion and applied to, for example, model predictive control. See [10] for such ideas.

\section{References}

[1] Stephen Boyd and Lieven Vandenberghe. Convex optimization. Course Reader for EE364, Introduction to Convex Optimization with Engineering Applications, Stanford University, May 3, 1999.

[2] Leo Breiman, Jerome H. Friedman, Richard A. Olshen, and Charles J. Stone. Classification And Regression Trees. Wadsworth \& Brooks/Cole Advanced Books \& Software, 1984. 
[3] J. Fan and I. Gijbels. Local Polynomial Modelling and Its Applications. Chapman \& Hall, 1996.

[4] Roderick Murray-Smith and Tor Arne Johansen, editors. Multiple Model Approaches to Modelling and Control. Taylor \& Francis, 1997.

[5] E. Nadaraya. On estimating regression. Theory of Probability and its Applications, 10:186-190, 1964.

[6] Jacob Roll, Alexander Nazin, and Lennart Ljung. A non-asymptotic approach to local modelling. In The 41st IEEE Conference on Decision and Control, December 2002.

[7] Jacob Roll, Alexander Nazin, and Lennart Ljung. Non-asymptotic optimal guaranteed MSE function estimation. Technical Report LiTH-ISY-R2415, Department of Electrical Engineering, Linköping University, SE-581 83 Linköping, Sweden, 2002.

[8] Jacob Roll, Alexander Nazin, and Lennart Ljung. Local modelling of nonlinear dynamic systems using direct weight optimization. Submitted to SYSID'03, August 2003.

[9] J. Sjöberg, Q. Zhang, L. Ljung, A. Benveniste, B. Delyon, P. Y. Glorennec, H. Hjalmarsson, and A. Juditsky. Nonlinear black-box modeling in system identification: a unified overview. Automatica, 31(12):1691-1724, 1995.

[10] Anders Stenman. Model on Demand: Algorithms, Analysis and Applications. PhD thesis, Department of Electrical Engineering, Linköping University, SE-581 83 Linköping, Sweden, 1999.

[11] G. Watson. Smooth regression analysis. Sankhyā, A(26):359-372, 1964. 